\title{
On weighted transplantation and multipliers for Laguerre expansions
}

\author{
Krzysztof Stempak ] and Walter Trebels
}

(April 22, 1993 version)

\begin{abstract}
Using the standard square-function method (based on the Poisson semigroup), multiplier conditions of Hörmander type are derived for Laguerre expansions in $L^{p}$-spaces with power weights in the $A_{p}$-range; this result can be interpreted as an "upper end point" multiplier criterion which is fairly good for $p$ near 1 or near $\infty$. A weighted generalization of Kanjin's [10] transplantation theorem allows to obtain a "lower end point" multiplier criterion whence by interpolation nearly "optimal" multiplier criteria (in dependance of $p$, the order of the Laguerre polynomial, the weight).
\end{abstract}

Key words. Laguerre polynomials, sufficient multiplier conditions, transplantation, fractional differences, weighted Lebesgue spaces

AMS(MOS) subject classifications. 42A45, 42B25, 42C10

\section{Introduction}

In the last fifteen years a considerable activity initiated by a series of papers of Markett has taken place to study the Harmonic Analysis for Laguerre expansions.

There are several ways of studying Laguerre expansions and corresponding multipliers. A principal one is based on the system $\left\{L_{k}^{\alpha}\right\}_{k=0}^{\infty}$ of Laguerre polynomials, see Szegö 25, p. 100], which are orthogonal in $L^{2}\left(\mathbf{R}_{+}, x^{\alpha} e^{-x} d x\right), \alpha>-1$. Weighted $L^{p}$-inequalities, proved for this system by Muckenhoupt [14, turn out to be very useful and imply $L^{p}$-estimates for Laguerre function expansions. However, as far as multipliers are concerned, this system is hard to deal with. For instance, Askey and Hirschman proved that uniform boundedness of Cesàro multiplier sequences of any positive order is restricted to $p=2$ only.

\footnotetext{
${ }^{1}$ Institute of Mathematics, University of Wrocław, Wrocław, Poland. The work of this author was done during a stay at the Fachbereich Mathematik of the TH Darmstadt and was supported in part by the TH Darmstadt and in part by the Deutsche Forschungsgemeinschaft under grant $436 \mathrm{POL} / 115 / 1 / 0$.

${ }^{2}$ Fachbereich Mathematik, TH Darmstadt, D-6100 Darmstadt, Germany.
} 
Another principal type of expansion was discussed by Görlich and Markett [8], [11]. To become more precise, let us introduce the Lebesgue spaces

$$
L_{v(\gamma)}^{p}=\left\{f:\|f\|_{L_{v(\gamma)}^{p}}=\left(\int_{0}^{\infty}|f(x)|^{p} x^{\gamma} d x\right)^{1 / p}<\infty\right\}, \quad 1 \leq p<\infty, \quad \gamma>-1 .
$$

If we define the Laguerre function system $\left\{l_{k}^{\alpha}\right\}$ by

$$
l_{k}^{\alpha}(x)=(k ! / \Gamma(k+\alpha+1))^{1 / 2} e^{-x / 2} L_{k}^{\alpha}(x), \quad \alpha>-1, \quad n \in \mathbf{N}_{0},
$$

then it is orthonormal in $L^{2}\left(\mathbf{R}_{+}, x^{\alpha} d x\right)$. If $\gamma<p(\alpha+1)-1$ we can associate to $f \in L_{v(\gamma)}^{p}$ the Laguerre series

$$
f(x) \sim \sum_{k=0}^{\infty} a_{k} l_{k}^{\alpha}(x), \quad a_{k}=\int_{0}^{\infty} f(x) l_{k}^{\alpha}(x) x^{\alpha} d x .
$$

Let $m=\left\{m_{k}\right\}$ be a sequence of real or complex numbers and associate to $m$ the operator

$$
T_{m} f(x)=\sum_{k=0}^{\infty} m_{k} a_{k} l_{k}^{\alpha}(x)
$$

for all $f$ of the type $f(x)=p(x) e^{-x / 2}, p$ polynomial (the set of these $f$ is dense in $L_{v(\gamma)}^{p}$, see [14]). The sequence $m$ is called a bounded multiplier on $L_{v(\gamma)}^{p}$, notation $m \in M_{\alpha, \gamma}^{p}$, if

$$
\|m\|_{M_{\alpha, \gamma}^{p}}:=\inf \left\{C:\left\|T_{m} f\right\|_{L_{v(\gamma)}^{p}} \leq C\|f\|_{L_{v(\gamma)}^{p}}\right\}
$$

is finite for all $f$ as before. We note the duality property $\left(1 / p+1 / p^{\prime}=1\right)$

$$
M_{\alpha, \gamma}^{p}=M_{\alpha, \alpha p^{\prime}-\gamma p^{\prime} / p}^{p^{\prime}}, \quad-1<\gamma<p(\alpha+1)-1, \quad 1<p<\infty .
$$

The purpose of this paper is to obtain a better insight into the structure of the Laguerre multiplier space $M_{\alpha, \gamma}^{p}$ from the point of view of sufficient conditions. We mention that Gasper and Trebels [7] discussed this question from the point of view of necessary conditions.

Remarks. 1) In [7] another scaling of the orthogonal system $\left\{l_{k}^{\alpha}\right\}$ is used, but it follows directly that our $M_{\alpha, \gamma}^{p}$-space coincides with the space $M_{w(\gamma)}^{p}$ in [7]. The $l_{k}^{\alpha}$ 's have the great advantage to possess a nice convolution structure as shown by Görlich and Markett [8].

2) The standard orthonormal system on $L^{2}\left(\mathbf{R}_{+}, d x\right)$ is given by

$$
\mathcal{L}_{k}^{\alpha}(x)=(k ! / \Gamma(k+\alpha+1))^{1 / 2} x^{\alpha / 2} e^{-x / 2} L_{k}^{\alpha}(x), \quad k \in \mathbf{N}_{0} .
$$

Though the Parseval identity follows at once, its disadvantage is to be seen in the fact that no nice convolution structure is available. If we denote by $\mathcal{M}_{\alpha, \gamma}^{p}$ the multiplier 
space with respect to the system $\left\{\mathcal{L}_{k}^{\alpha}\right\}$ (introduced analogously to $M_{\alpha, \gamma}^{p}$ ), then one can easily show the following connection between the two multiplier spaces

$$
\mathcal{M}_{\alpha, \gamma}^{p}=M_{\alpha, \gamma+\alpha p / 2}^{p}
$$

3) Markett [11] and Thangavelu [24] considered another orthonormalized system on $L^{2}\left(\mathbf{R}_{+}, d x\right)$, namely $\left\{\varphi_{k}^{\alpha}\right\}$,

$$
\varphi_{k}^{\alpha}(x)=\mathcal{L}_{k}^{\alpha}\left(x^{2}\right)(2 x)^{1 / 2}, \quad k \in \mathbf{N}_{0} .
$$

Again one makes sure that the associated multiplier space coincides with the previously introduced $M_{\alpha, \gamma+\alpha p / 2+p / 4-1 / 2}^{p}$. Thus, if one wants to study multipliers with respect to these three orthonormal systems, one only needs to discuss $M_{\alpha, \gamma}^{p}$ for various parameters $\gamma$.

The sufficient multiplier conditions we have in mind are described in terms of the following "weak-bounded-variation" sequence spaces from Gasper and Trebels [4]

$$
w b v_{q, s}=\left\{m \in l^{\infty}:\|m\|_{q, s}<\infty\right\}, \quad 1 \leq q \leq \infty, \quad s>0,
$$

where the norm on this sequence space is given by

$$
\|m\|_{q, s}=\|m\|_{\infty}+\sup _{n}\left(\sum_{k=n}^{2 n} k^{-1}\left|k^{s} \Delta^{s} m_{k}\right|^{q}\right)^{1 / q}
$$

(with the standard interpretation for $q=\infty$ ) and the difference operator $\Delta^{s}$ of fractional order $s$ by

$$
\Delta^{s} m_{k}=\sum_{j=0}^{\infty} A_{j}^{-s-1} m_{k+j}
$$

whenever the sum converges. We are now ready to formulate a first sufficient multiplier criterion for Laguerre expansions (to be proved in Section 3).

Theorem 1.1 Let $\alpha \geq 0,1<p<\infty$, and $m \in w b v_{2, s}$ for some $s>\alpha+1$. Then $m \in M_{\alpha, \gamma}^{p}$ and $\|m\|_{M_{\alpha, \gamma}^{p}} \leq C\|m\|_{2, s}$, provided

$$
(\alpha+1) \max \{-p / 2,-1\}<\gamma-\alpha<(\alpha+1) \min \{p / 2, p-1\}
$$

i.e., under the above assumptions there holds in the sense of continuous embedding

$$
w b v_{2, s} \subset M_{\alpha, \gamma}^{p}
$$


Remarks. 1) For $\gamma=\alpha$ Theorem 1.1 coincides with an unpublished result of Dietrich, Görlich, Hinsen, and Markett (due to a written communication of C. Markett). For integer $\alpha=n$, a weaker version of Theorem 1.1, namely

$$
w b v_{\infty, s(n)} \subset M_{n, n}^{p}, \quad s(n)= \begin{cases}n+2 & \text { odd } n \\ n+3 & \text { even } n\end{cases}
$$

can be read off from Thangavelu [23].

2) By the weighted transplantation theorem to be deduced in Section 4 Theorem 1.1 implies

$$
w b v_{2, s_{0}} \subset M_{\alpha, \alpha}^{p}, \quad s_{0}>1, \quad \alpha \geq 0, \quad \frac{2 \alpha+2}{\alpha+2}<p<\frac{2 \alpha+2}{\alpha} .
$$

Following the lines of Connett and Schwartz [2], interpolation between Theorem 1.1 for $\gamma=\alpha$ with $p$ near 1 and (5) leads to Part a) of

Corollary 1.2 a) Let $\alpha \geq 0$ and $1<p<\infty$, then

$$
w b v_{2, s} \subset M_{\alpha, \alpha}^{p}, \quad s>\max \left\{s_{c}(p), 1\right\},
$$

where the quantity $s_{c}(p)=(2 \alpha+2)|1 / p-1 / 2|$ plays the role of a critical index in the multiplier space $M_{\alpha, \alpha}^{p}$.

b) Let $\alpha>-1 / 3$ and $1 \leq p<2$, then

$$
M_{\alpha, \alpha}^{p} \subset w b v_{p^{\prime}, s^{\prime}}, \quad 0<s^{\prime} \leq s_{c}(p)-4(1 / p-1 / 2) / 3 .
$$

Part b) is a slight extension of a result in Gasper and Trebels [7]. The corollary nicely shows where Laguerre multipliers live. Surprising is the smoothness gap of size $4|1 / p-1 / 2| / 3$, since in other settings a gap of size $|1 / p-1 / 2|$ is well known, see e.g. [6] in the Jacobi case $(\alpha,-1 / 2)$.

If $s_{c}(p) \geq 1$ the result in Part a) is best possible in the following sense (continuous embedding)

$$
w b v_{2, s^{\prime}} \not \subset M_{\alpha, \alpha}^{p}, \quad s^{\prime}<s_{c}(p),
$$

as the example of the Cesàro means $m_{n, \nu}$ of order $\nu$ shows: observing

$$
m_{n, \nu}(k)=\left\{\begin{array}{ll}
A_{n-k}^{\nu} / A_{n}^{\nu} & , 0 \leq k \leq n \\
0 & , k>n
\end{array}, \quad \Delta^{s^{\prime}} m_{n, \nu}(k)=\left\{\begin{array}{ll}
A_{n-k}^{\nu-s^{\prime}} / A_{n}^{\nu} & , 0 \leq k \leq n \\
0 & , k>n
\end{array},\right.\right.
$$

it immediately follows that $m_{n, \nu} \in w b v_{2, s^{\prime}}$ uniformly in $n$ if $s^{\prime}<\nu+1 / 2<s_{c}(p)$. But it is well known that $\left\|m_{n, \nu}\right\|_{M_{\alpha, \alpha}^{p}} \geq C(n+1)^{s_{c}(p)-\nu-1 / 2}$ (see e.g. [12]).

On the other hand concerning the result in Part b) it is shown in [7] that at least for $p=1$ and $\alpha \geq 0$ the necessary condition is best possible. In [7, II] there is given 
a modification of the $w b v$-condition (not nicely comparable in the $w b v$-framework) which suggests a smoothness gap of size $|1 / p-1 / 2|$ if one could modify in the same sense the sufficient condition.

The plan of the paper is as follows. In Section 2 we develop the required squarefunction calculus based on the Poisson kernel. In Section 3 we give the relevant estimates for the Poisson means of the multiplier which allow one to use the standard square-function method (see e.g. [2]). In Section 4 we turn to a generalization of Kanjin's [10] transplantation theorem, thus including Thangavelu's [24] modification, by admitting more general power weights. This allows some further insight into the structure of Laguerre multipliers.

Acknowledgements. Some of the ideas occurring in this paper were developed in other work done jointly by George Gasper and the second author. At this point we express our indebtedness to these discussions. The first author thanks the Fachbereich Mathematik of the TH Darmstadt for hospitality and financial support.

\section{On square-functions for Laguerre expansions}

The main tool we will use is the twisted generalized convolution defined in $L^{1}\left(\mathbf{R}_{+}, d \mu_{\alpha}\right), d \mu_{\alpha}(x)=$ $x^{2 \alpha+1} d x, \alpha \geq 0$, by

$$
f \times g(x)=\int_{0}^{\infty} \tau_{x} f(y) g(y) d \mu_{\alpha}(y)
$$

where the twisted generalized translation operator $\tau_{x}$ is given by

$$
\tau_{x} f(y)=\frac{\Gamma(\alpha+1)}{\pi^{1 / 2} \Gamma(\alpha+1 / 2)} \int_{0}^{\pi} f\left((x, y)_{\theta}\right) \mathcal{J}_{\alpha-1 / 2}(x y \sin \theta)(\sin \theta)^{2 \alpha} d \theta
$$

$\mathcal{J}_{\beta}(x)=\Gamma(\beta+1) J_{\beta}(x) /(x / 2)^{\beta}, J_{\beta}$ denoting the Bessel function of order $\beta>-1$, and

$$
(x, y)_{\theta}=\left(x^{2}+y^{2}-2 x y \cos \theta\right)^{1 / 2}
$$

this convolution is commutative - for all this see Görlich and Markett [8] and Stempak [20]. For the following it is convenient to work with the transformed system

$$
\psi_{k}^{\alpha}(x)=(2 k ! / \Gamma(k+\alpha+1))^{1 / 2} e^{-x^{2} / 2} L_{k}^{\alpha}\left(x^{2}\right), \quad k \in \mathbf{N}_{0},
$$

which, on account of the orthonormality of $\left\{l_{k}^{\alpha}\right\}$, is obviously orthonormal on $L^{2}\left(\mathbf{R}_{+}, d \mu_{\alpha}\right)$. We introduce the norm

$$
\|f\|_{p, \delta}=\left(\int_{0}^{\infty}|f(x)|^{p} x^{2 \delta} d \mu_{\alpha}(x)\right)^{1 / p}
$$


this will not lead to any confusion with the $w b v$-norm. We note that the $\psi_{k}^{\alpha}$ 's are eigenfunctions, with eigenvalues $\lambda_{k}$, of the positive symmetric in $L^{2}\left(\mathbf{R}_{+}, d \mu_{\alpha}\right)$ differential operator $L$,

$$
L=-\left(\frac{d^{2}}{d x^{2}}+\frac{2 \alpha+1}{x} \frac{d}{d x}-x^{2}\right), \quad \lambda_{k}=4 k+2 \alpha+2 .
$$

We mention the following transform property of the twisted generalized convolution with respect to $\psi_{k}^{\alpha}$-Laguerre expansions; if $f \sim \sum c_{k} \psi_{k}^{\alpha}$ and $f \times g \sim \sum c_{k} d_{k} \psi_{k}^{\alpha}$, then $g(x) \sim \Gamma(\alpha+1) \sum d_{k} L_{k}^{\alpha}\left(y^{2}\right) e^{-y^{2} / 2}$. To $f \sim \sum c_{k} \psi_{k}^{\alpha}$ we associate its Poisson means

$$
P^{t} f=\sum_{k=0}^{\infty} \exp \left(-t \lambda_{k}\right) c_{k} \psi_{k}^{\alpha}=f \times p_{t}, \quad t>0
$$

with Poisson kernel $p_{t}(y)=c_{\alpha} \sum \exp \left(-t \lambda_{k}\right) L_{k}^{\alpha}\left(y^{2}\right) e^{-y^{2} / 2}$, and the square-function

$$
g_{1}(f)^{2}(x)=\int_{0}^{\infty}\left|\frac{\partial}{\partial t} P^{t} f(x)\right|^{2} t d t=\int_{0}^{\infty}\left|\sum_{k=0}^{\infty} \lambda_{k} \exp \left(-t \lambda_{k}\right) c_{k} \psi_{k}^{\alpha}(x)\right|^{2} t d t
$$

for appropriate $f$. Since for $\alpha \geq 0$ the semigroup $\left\{P^{t}\right\}_{t>0}$ forms a positive contraction semigroup (see [8] and [21]), by the Coifman, Rochberg, and Weiss refinement of Stein's general Littlewood-Paley theory (see Meda [13])

$$
C^{-1}\|f\|_{p, 0} \leq\left\|g_{1}(f)\right\|_{p, 0} \leq C\|f\|_{p, 0}, \quad 1<p<\infty
$$

is true. We want to extend (7) to the weighted case $\delta \neq 0$.

Proposition 2.1 Let $1<p<\infty, \alpha \geq 0$, and $-(\alpha+1)<\delta<(\alpha+1)(p-1)$. Then

$$
C^{-1}\|f\|_{p, \delta} \leq\left\|g_{1}(f)\right\|_{p, \delta} \leq C\|f\|_{p, \delta}
$$

Proof. Assume for the moment that the right inequality holds for the $\delta$ 's indicated. We show that then the left one follows. Since by straightforward calculation, based on Parseval's identity, $\|f\|_{2,0}=C(\alpha)\left\|g_{1}(f)\right\|_{2,0}$, polarization and Hölder's inequality give

$$
\begin{aligned}
\left|\int_{0}^{\infty} f_{1}(x) f_{2}(x) d \mu_{\alpha}(x)\right| & \leq C \int_{0}^{\infty} g_{1}\left(f_{1}\right)(x) x^{2 \delta / p} x^{-2 \delta / p} g_{1}\left(f_{2}\right)(x) d \mu_{\alpha}(x) \\
& \leq C\left\|g_{1}\left(f_{1}\right)\right\|_{p, \delta}\left\|g_{1}\left(f_{2}\right)\right\|_{p^{\prime},-\delta p^{\prime} / p}
\end{aligned}
$$

Setting $f_{2}(x)=x^{2 \delta / p} h(x)$ and using the right hand inequality in Proposition 2.1 one obtains

$$
\left|\int_{0}^{\infty} f_{1}(x) x^{2 \delta / p} h(x) d \mu_{\alpha}(x)\right| \leq C\left\|g_{1}\left(f_{1}\right)\right\|_{p, \delta}\|h\|_{p^{\prime}, 0}
$$


for all $h \in L^{p^{\prime}}\left(d \mu_{\alpha}\right)$ and $-(\alpha+1)<-2 \delta p^{\prime} / p<(\alpha+1)\left(p^{\prime}-1\right)$ so that finally the converse of Hölder's inequality gives the desired left hand side inequality

$$
\left\|f_{1}\right\|_{p, \delta}=\left\|f_{1} x^{2 \delta / p}\right\|_{p, 0} \leq C\left\|g_{1}\left(f_{1}\right)\right\|_{p, \delta} .
$$

To extend the right hand side inequality of (7) to the weighted case $\delta \neq 0$ we adapt an approach of Stein [17]. The following is a variation of a corresponding remark in [18, p. 271].

Lemma 2.2 For $\alpha \geq 0$ let $K(x, y)$ be a homogeneous kernel, $K(\lambda x, \lambda y)=\lambda^{-(2 \alpha+2)} K(x, y)$, satisfying

$$
\int_{0}^{\infty}|K(1, y)| y^{-(2 \alpha+2) / p} d \mu_{\alpha}(y)<\infty
$$

Then the operator

$$
T f(x)=\int_{0}^{\infty} K(x, y) f(y) d \mu_{\alpha}(y)
$$

is bounded on $L^{p}\left(d \mu_{\alpha}\right)$.

The particular kernel in the next lemma is the key of the desired extension.

Lemma 2.3 For $\alpha \geq 0$ the kernel

$$
K(x, y)=\left|1-(x / y)^{2 \delta / p}\right| \int_{0}^{\pi}(x, y)_{\theta}^{-(2 \alpha+2)}(\sin \theta)^{2 \alpha} d \theta
$$

satisfies the properties of Lemma 2.2 provided $-(\alpha+1)<\delta<(\alpha+1)(p-1)$.

Proof. The homogeneity property of the kernel is clear. For the second property note that there are three singularities: $0, \infty$, and 1 and the required integrability of $K(1, y)$ follows once we show that

$$
\int_{0}^{\pi}(1, y)_{\theta}^{-(2 \alpha+2)}(\sin \theta)^{2 \alpha} d \theta \leq C \begin{cases}1 & \text { as } y \rightarrow 0 \\ |1-y|^{-1} & \text { as } y \rightarrow 1 \\ y^{-(2 \alpha+2)} & \text { as } y \rightarrow \infty\end{cases}
$$

For small $y$ note that $(1, y)_{\theta} \approx 1,0<\theta<\pi$ whereas $(1, y)_{\theta} \approx y$ for large $y$. Finally, for $y \approx 1$ it follows that

$$
\begin{gathered}
\int_{0}^{\pi} \frac{(\sin \theta)^{2 \alpha}}{\left((1-y)^{2}+4 y \sin ^{2}(\theta / 2)\right)^{\alpha+1}} d \theta \leq C|1-y|^{-(2 \alpha+2)} \int_{0}^{|1-y|}(\sin \theta)^{2 \alpha} d \theta \\
+C \int_{|1-y|}^{\pi} \sin ^{-2}(\theta / 2) d \theta \leq C|1-y|^{-1}
\end{gathered}
$$

so that (8) is obvious.

The modification to twisted generalized convolution operators of Stein's [17 result now reads 
Lemma 2.4 Suppose $|A(x)| \leq C x^{-(2 \alpha+2)}$ and $T f=A \times f$ is bounded on $L^{p}\left(d \mu_{\alpha}\right), 1<$ $p<\infty$. Then we also have the weighted inequality

$$
\|T f\|_{p, \delta} \leq C\|f\|_{p, \delta}, \quad-(\alpha+1)<\delta<(\alpha+1)(p-1) .
$$

The proof follows along the pattern of [17]. We only note that

$$
x^{2 \delta / p} A \times f(x)=A \times\left(y^{2 \delta / p} f\right)(x)-\int_{0}^{\infty}\left(1-(x / y)^{2 \delta / p}\right) \tau_{x} A(y) f(y) y^{2 \delta / p} d \mu_{\alpha}(y)
$$

and, since $\left|\mathcal{J}_{\alpha-1 / 2}(t)\right| \leq 1, \alpha \geq 0$, the last integral can be estimated by

$$
\int_{0}^{\infty} K(x, y)|f(y)| y^{2 \delta / p} d \mu_{\alpha}(y)
$$

where $K$ is the kernel from Lemma 2.3, hence the assertion by Lemma 2.2.

The above scalar-valued result can be extended to the case of functions taking their values in a Hilbert space, see [18, pp. 45], by a repetition of the arguments given for the scalar-valued case.

Proposition 2.5 Let $A(x)$ be a function on $\mathbf{R}_{+}$taking values in $B\left(\mathcal{H}_{1}, \mathcal{H}_{2}\right), \mathcal{H}_{i}$ being separable Hilbert spaces, and satisfying $\|A\| \leq C x^{-(2 \alpha+2)}$. Further, if one defines $T f=A \times f$ for $f \in L^{p}\left(\mathbf{R}_{+}, \mathcal{H}_{1}\right)$ and if

$$
\int_{0}^{\infty}\|T f(x)\|_{\mathcal{H}_{2}}^{p} x^{2 \delta} d \mu_{\alpha}(x) \leq C \int_{0}^{\infty}\|f(x)\|_{\mathcal{H}_{1}}^{p} x^{2 \delta} d \mu_{\alpha}(x), \quad 1<p<\infty
$$

holds for $\delta=0$, then the same inequality is valid for all $\delta$ 's satisfying $-(\alpha+1)<\delta<$ $(\alpha+1)(p-1)$.

To complete the proof of Proposition 2.1 we choose in the preceding proposition $\mathcal{H}_{1}=\mathbf{C}, \mathcal{H}_{2}=L^{2}\left(\mathbf{R}_{+}, t d t\right)$ and $T f=(\partial / \partial t) P^{t} f=f \times(\partial / \partial t) p_{t}$. It is shown in Thangavelu [23, Lemma 3.1] that

$$
\left\|(\partial / \partial t) p_{t}(x)\right\|_{L^{2}\left(\mathbf{R}_{+}, t d t\right)} \leq C x^{-(2 \alpha+2)}
$$

where $(\partial / \partial t) p_{t}=c_{\alpha} \sum \lambda_{k} \exp \left(-t \lambda_{k}\right) L_{k}^{\alpha}\left(y^{2}\right) e^{-y^{2} / 2}$. Since (7) holds, all hypotheses of Proposition 2.5 are satisfied and hence Proposition 2.1 is established.

For the proof of the multiplier Theorem 1.1 we need the standard variations on the $g_{1}$-function, namely $g_{\sigma}$-functions and a $g_{\lambda}^{*}$-function.

We note that a substitution in (6) gives

$$
g_{1}(f)^{2}(x)=\int_{0}^{1}\left|\sum_{k=0}^{\infty} \lambda_{k} r^{\lambda_{k}} c_{k} \psi_{k}^{\alpha}(x)\right|^{2}|\log r| \frac{d r}{r} .
$$


Denoting $u(x, r)=P^{t} f(x), e^{-t}=r$, and following Strichartz [22] we introduce as $\sigma$-th derivative of $u(x, r)$

$$
d_{\sigma} u(x, r)=\sum_{k=0}^{\infty} \lambda_{k}^{\sigma} r^{\lambda_{k}} c_{k} \psi_{k}^{\alpha}(x)
$$

and set

$$
g_{\sigma}(f)^{2}(x)=\int_{0}^{1}\left|d_{\sigma} u(x, r)\right|^{2}|\log r|^{2 \sigma-1} \frac{d r}{r} .
$$

For the definiton of the $g_{\lambda}^{*}$-function we need a generalized Euclidean translation (occurring in the framework of modified Hankel transforms, cf. [20])

$$
\tau_{x}^{E} f(y)=\frac{\Gamma(\alpha+1)}{\pi^{1 / 2} \Gamma(\alpha+1 / 2)} \int_{0}^{\pi} f\left((x, y)_{\theta}\right)(\sin \theta)^{2 \alpha} d \theta
$$

and its associated convolution

$$
f * g(x)=\int_{0}^{\infty} \tau_{x}^{E} f(y) g(y) d \mu_{\alpha}(y)
$$

then the $g_{\lambda}^{*}$-function is defined by

$$
g_{\lambda}^{*}(f)^{2}(x)=\int_{0}^{1} K_{|\log r|} *\left|d_{1} u(\cdot, r)\right|^{2}(x)|\log r| \frac{d r}{r}
$$

where $K_{t}(y)=\delta_{\sqrt{t}} K(y), K(y)=\left(1+y^{2}\right)^{-\lambda}$ and $\delta_{u} f(y)=u^{-2(\alpha+1)} f(y / u)$ is an $L^{1}\left(d \mu_{\alpha}\right)$-invariant dilation.

\section{Proposition 2.6 a)}

$$
g_{\rho}(f)(x) \leq C g_{\sigma}(f)(x) \quad \text { a.e., } 1 \leq \rho \leq \sigma
$$

for all $f \in L^{p}\left(x^{2 \delta} d \mu_{\alpha}\right)$ for which the right hand side makes sense.

b)

$$
\left\|g_{\lambda}^{*}(f)\right\|_{p, \delta} \leq C\|f\|_{p, \delta}, \quad \lambda>\alpha+1, \quad p \geq 2
$$

provided $-(\alpha+1)<\delta<p(\alpha+1)(1 / 2-1 / p)$.

Proof. Assertion a) is proved just as in Strichartz [22]. For the proof of b) we note that the method in Stein [18, p. 91] works. Start with the basic inequality ( $M$ denotes the Hardy-Littlewood maximal operator in the homogeneous space $\left(\mathbf{R}_{+}, d \mu_{\alpha}, \rho\right)$ where $\rho$ is the usual distance on $\mathbf{R}_{+}$).

$$
\int_{0}^{\infty} g_{\lambda}^{*}(f)^{2}(x) h(x) d \mu_{\alpha} \leq C \int_{0}^{\infty} g_{1}(f)^{2}(x) M h(x) d \mu_{\alpha}
$$


which also holds in the present setting on account of the formulae (3.6), (3.8) and (3.13) in Stempak [20]. Here the assumption $\lambda>\alpha+1$ implies $K(y) \in L^{1}\left(d \mu_{\alpha}\right)$.

In the case $p=2$ choose $h(x)=x^{2 \delta}$ in (10) and note that the maximal function $M h(x)=C x^{2 \delta} M h(1)$ for $-(\alpha+1)<\delta \leq 0$, hence the assertion by Proposition 2.1. In the case $q=p / 2>1$ choose $h(x)=x^{4 \delta / p} h_{1}(x)$ in (10) and apply Hölder's inequality $\left(1 / q+1 / q^{\prime}=1\right)$ to obtain

$$
\begin{gathered}
\int_{0}^{\infty} g_{\lambda}^{*}(f)^{2}(x) x^{4 \delta / p} h_{1}(x) d \mu_{\alpha} \leq C \int_{0}^{\infty} g_{1}(f)^{2}(x) x^{4 \delta / p} x^{-4 \delta / p} M\left(h_{1} x^{4 \delta / p}\right) d \mu_{\alpha} \\
\leq C\left(\int_{0}^{\infty} g_{1}(f)^{p}(x) x^{2 \delta} d \mu_{\alpha}\right)^{2 / p}\left(\int_{0}^{\infty} M\left(h_{1} x^{4 \delta / p}\right)^{q^{\prime}}(x) x^{-4 q^{\prime} \delta / p} d \mu_{\alpha}\right)^{1 / q^{\prime}} \\
\leq C\left\|g_{1}(f)\right\|_{p, \delta}^{2}\left\|h_{1}\right\|_{q^{\prime}, 0}
\end{gathered}
$$

for $x^{-4 q^{\prime} \delta / p} \in A_{q^{\prime}}\left(d \mu_{\alpha}\right)$ by the assumption on $\delta$ (see [20, II]). Taking the supremum over all $h_{1},\left\|h_{1}\right\|_{q^{\prime}, 0} \leq 1$ gives

$$
\left\|g_{\lambda}^{*}(f)\right\|_{p, \delta}^{2}=\left\|g_{\lambda}^{*}(f) x^{4 \delta / p}\right\|_{q, 0} \leq C\left\|g_{1}(f)\right\|_{p, \delta}^{2} \leq C\|f\|_{p, \delta}^{2} .
$$

\section{Proof of Theorem 1.1.}

Since we follow the standard method (see e.g. [2, p.73]), we only indicate the main steps. We use the notation

$$
f \sim \sum c_{k} \psi_{k}^{\alpha}, \quad S_{m} f \sim \sum m_{k} c_{k} \psi_{k}^{\alpha}
$$

and work on a dense subset of $L^{p}\left(x^{2 \delta} d \mu_{\alpha}\right)$ (see [14]) such that we can write $=$ instead of $\sim$ in the preceding formulae. We note that the multiplier space associated to $\|\cdot\|_{p, \delta}$ coincides with $M_{\alpha, \alpha+\delta}^{p}$. Thus all we need to show, under the assumptions of Theorem 1.1, is

$$
\left\|S_{m} f\right\|_{p, \delta} \leq C\|m\|_{2, s}\|f\|_{p, \delta}, \quad \delta=\gamma-\alpha .
$$

If we assume for the moment that

$$
g_{s+1}\left(S_{m} f\right)(x) \leq C\|m\|_{2, s} g_{s}^{*}(f)(x) \quad \text { a.e. }
$$

holds, then (11) is proved in the case $p \geq 2,-(\alpha+1)<\delta<p(\alpha+1)(1 / 2-1 / p)$ by the following chain of norm inequalities if we choose $\lambda=s>\alpha+1$ in Proposition 2.6 b)

$$
\begin{gathered}
\left\|S_{m} f\right\|_{p, \delta} \leq C\left\|g_{1}\left(S_{m} f\right)\right\|_{p, \delta} \leq C\left\|g_{s+1}\left(S_{m} f\right)\right\|_{p, \delta} \\
\leq C\|m\|_{2, s}\left\|g_{s}^{*}(f)\right\|_{p, \delta} \leq C\|m\|_{2, s}\|f\|_{p, \delta} .
\end{gathered}
$$


In the case $p=2$ the result (11) extends at once to $-(\alpha+1)<\delta<\alpha+1$ by duality (1). Repeating the interpolation and duality arguments in Hirschman [9, p.50] yields Theorem 1.1 provided (12) holds.

Let us turn to the proof of (12). First we note that (up to a constant) we don't change the $g_{\sigma}$-function (9) if we substitute $r^{2}$ for $r$. By the properties of twisted convolution we have

$$
d_{s+1} S_{m} u\left(x, r^{2}\right)=d_{s} M(\cdot, r) \times d_{1} u(\cdot, r)(x)=\int_{0}^{\infty} \tau_{x} d_{1} u(y, r) d_{s} M(y, r) d \mu_{\alpha}(y),
$$

where

$$
d_{s} M(y, r)=c_{\alpha} \sum_{k=0}^{\infty} \lambda_{k}^{s} m_{k} r^{\lambda_{k}} L_{k}^{\alpha}\left(y^{2}\right) e^{-y^{2} / 2} .
$$

Basic properties of $d_{s} M(y, r)$, we need for the proof of (12), are contained in

Proposition 3.1 Let $\alpha \geq 0$ and $m \in w b v_{2, s}$ for $s>\alpha+1$. Then

a)

$$
\sup _{y}\left|d_{s} M(y, r)\right| \leq C r^{2 \alpha+2}(1-r)^{-s-\alpha-1}\|m\|_{\infty}
$$

b)

$$
\int_{0}^{\infty}\left|y^{s} d_{s} M(y, r)\right|^{2} d \mu_{\alpha}(y) \leq C r^{4 \alpha+4}(1-r)^{-s-\alpha-1}\|m\|_{2, s}^{2} .
$$

Suppose that Proposition 3.1 is proved, then one obtains by the Cauchy-Schwarz inequality

$$
\begin{aligned}
g_{s+1}\left(S_{m} f\right)^{2}(x)=\int_{0}^{1}\left|\left(\int_{0}^{\sqrt{1-r}}+\int_{\sqrt{1-r}}^{\infty}\right) \tau_{x} d_{1} u(y, r) d_{s} M(y, r) d \mu_{\alpha}(y)\right|^{2}|\log r|^{2 s+1} \frac{d r}{r} \\
\leq C\|m\|_{\infty}^{2} \int_{0}^{1} \frac{r^{4 \alpha+4}}{(1-r)^{\alpha+2 s+1}} \int_{0}^{\sqrt{1-r}}\left|\tau_{x} d_{1} u(y, r)\right|^{2} d \mu_{\alpha}(y)|\log r|^{2 s+1} \frac{d r}{r} \\
+C\|m\|_{2, s}^{2} \int_{0}^{1} \frac{r^{4 \alpha+4}}{(1-r)^{\alpha+s+1}} \int_{\sqrt{1-r}}^{\infty}\left|y^{-s} \tau_{x} d_{1} u(y, r)\right|^{2} d \mu_{\alpha}(y)|\log r|^{2 s+1} \frac{d r}{r} \\
\leq C\|m\|_{\infty}^{2} \int_{0}^{1} \frac{r^{4 \alpha+4}|\log r|^{2 s}}{(1-r)^{\alpha+2 s+1}} \int_{0}^{\sqrt{1-r}} \tau_{x}^{E}\left(\left|d_{1} u(y, r)\right|^{2}\right) d \mu_{\alpha}(y)|\log r| \frac{d r}{r} \\
+C\|m\|_{2, s}^{2} \int_{0}^{1} \frac{r^{4 \alpha+4}|\log r|^{2 s}}{(1-r)^{\alpha+s+1}} \int_{\sqrt{1-r}}^{\infty} y^{-2 s} \tau_{x}^{E}\left(\left|d_{1} u(y, r)\right|^{2}\right) d \mu_{\alpha}(y)|\log r| \frac{d r}{r}
\end{aligned}
$$


because $\left|\tau_{x} f(y)\right| \leq \tau_{x}^{E}(|f|)(y)$, see [20]. Now we use in the first integral $y^{2} /|\log r| \leq 1$ if $0<y<\sqrt{1-r}$ and $r^{4}(|\log r| /(1-r))^{2 s+\alpha+1} \leq C$ for $0<r<1$, and in the second $r|\log r|^{s} y^{-2 s} \leq C\left(1+y^{2} /|\log r|\right)^{-s}$ if $1-r<y^{2}$ and arrive at

$$
\begin{aligned}
g_{s+1}\left(S_{m} f\right)^{2}(x) & \leq C\left\{\|m\|_{\infty}^{2}+\|m\|_{2, s}^{2}\right\} \int_{0}^{1} K_{|\log r|} *\left|d_{1} u(\cdot, r)\right|^{2}(x)|\log r| \frac{d r}{r} \\
& \leq C\|m\|_{2, s}^{2} g_{s}^{*}(f)^{2}(x)
\end{aligned}
$$

by the definition of the $w b v$-norm.

Thus there remains only to prove Proposition 3.1. On account of [11, Lemma 1], 5th case, there holds

$$
\sup _{y}\left|L_{k}^{\alpha}\left(y^{2}\right) e^{-y^{2} / 2}\right| \leq C(k+1)^{\alpha}
$$

and hence Part a)

$$
\sup _{y}\left|d_{s} M(y, r)\right| \leq C\|m\|_{\infty} r^{2 \alpha+2} \sum_{k=0}^{\infty}(k+1)^{s+\alpha} r^{4 k} \leq C\|m\|_{\infty} r^{2 \alpha+2}(1-r)^{-s-\alpha-1},
$$

since $\sum(k+1)^{\gamma} r^{k} \leq C(1-r)^{-\gamma-1}$ is true for $\gamma>-1$.

To prove Part b) we use a weighted Parseval formula. First we note that the coefficients $c_{k}$ in the $\psi_{k}^{\alpha}$-expansion are related to the Fourier Laguerre coefficients $\hat{g}_{\alpha}(k)$ of [7] in the following way

$$
c_{k}=\Gamma(\alpha+1)(\Gamma(k+\alpha+1) / 2 k !)^{1 / 2}\left(e^{y / 2} f\left(y^{1 / 2}\right)\right)_{\alpha}{ }^{\wedge}(k), \quad k \in \mathbf{N}_{0}
$$

then it follows from Gasper and Trebels [0], I], formulae (3) and (5) there, that

$$
\sum_{k=0}^{\infty} A_{k}^{\alpha+s}\left|\Delta^{s}\left(c_{k} \sqrt{k !} / \sqrt{\Gamma(k+\alpha+1)}\right)\right|^{2} \approx \int_{0}^{\infty}|f(x)|^{2} x^{2 \alpha+2 s+1} d x
$$

hence for the particular case $f=d_{s} M(\cdot, r)$

$$
\int_{0}^{\infty}\left|y^{s} d_{s} M(y, r)\right|^{2} d \mu_{\alpha}(y) \leq C \sum_{k=0}^{\infty} A_{k}^{\alpha+s}\left|\Delta^{s}\left(\lambda_{k}^{s} m_{k} r^{\lambda_{k}}\right)\right|^{2}=: I .
$$

We have to dominate $I$. Since similar computations for integer $s$ are contained in [2, p. 69] we only sketch the proof in that case. First note that $\Delta^{\kappa} r^{k}=(1-r)^{\kappa} r^{k}, \kappa>0$, and that

$$
\left|\Delta^{j}\left(\lambda_{k}^{s} r^{4 k}\right)\right|^{2} \leq C \sum_{i=0}^{j}(1-r)^{2 i} r^{8 k}(k+1)^{2(s-i-j)} ;
$$

then use these formulae in Leibniz' formula for differences

$$
I \leq C r^{4 \alpha+4} \sum_{j=0}^{s} \sum_{k=0}^{\infty} A_{k}^{\alpha+s}\left|\Delta^{j} m_{k}\right|^{2}\left|\Delta^{s-j}\left(\lambda_{k+j}^{s} r^{4 k+4 j}\right)\right|^{2}
$$




$$
\leq C r^{4 \alpha+4} \sum_{j=0}^{s}\|m\|_{2, j}^{2}(1-r)^{-\alpha-s-1} \leq C r^{4 \alpha+4}\|m\|_{2, s}^{2}(1-r)^{-\alpha-s-1}
$$

by the embedding properties of the $w b v$-spaces, see 4 .

If $s$ is strictly fractional, similar computations have been carried through in the proof of [5, Lemma 1] and again we only sketch the proof. We use Peyerimhoff's [15] version of Leibniz' formula for fractional differences which in our instance reads:

Let $s=[s]+\kappa, 0<\kappa<1$. Then

$$
\begin{gathered}
\Delta^{s}\left(\lambda_{k}^{s} r^{\lambda_{k}} m_{k}\right)=r^{2 \alpha+2} \sum_{i=0}^{[s]}\left(\begin{array}{c}
s \\
i
\end{array}\right) \Delta^{i}\left(\lambda_{k}^{s} r^{4 k}\right) \Delta^{s-i} m_{k+i}+r^{2 \alpha+2} m_{k} \Delta^{s}\left(\lambda_{k}^{s} r^{4 k}\right) \\
+(-1)^{[s]} r^{2 \alpha+2} R_{k},
\end{gathered}
$$

where the remainder term $R_{k}$ is given by

$$
\sum_{i=k+1+[s]}^{\infty} A_{i-k}^{-s-1}\left(m_{i}-m_{k}\right) \sum_{j=k+1}^{i-[s]} A_{i-[s]-j}^{-[s]-1}\left\{\Delta^{[s]}\left(\lambda_{j}^{s} r^{4 j}\right)-\Delta^{[s]}\left(\lambda_{k}^{s} r^{4 k}\right)\right\}
$$

Up to the terms which contain $\Delta^{s-i} m_{k+i}$ no smoothness of the sequence $m$ is required and in that case $\left|m_{k}\right|$ can be crudely estimated by $\|m\|_{\infty}$. To give an idea of the type of analysis required let us look at

$$
\begin{aligned}
& \left|\Delta^{s}\left(\lambda_{k}^{s} r^{4 k}\right)\right| \leq C \Delta^{s-[s+1]} \sum_{l=0}^{[s+1]}\left|\Delta^{l} r^{4 k}\right|\left|\Delta^{[s+1]-l} \lambda_{k}^{s}\right| \\
& \leq C \sum_{l=0}^{[s+1]}(1-r)^{l}\left(\sum_{i=0}^{k}+\sum_{i=k+1}^{\infty}\right) A_{i}^{[s]-s} r^{4(k+i)}(k+i+1)^{s-[s+1]+l} .
\end{aligned}
$$

For $0 \leq i \leq k$ one has $(k+i+1) \approx(k+1)$ and thus $\sum_{0}^{k} \ldots \leq C r^{4 k}(k+1)^{l-1}$; for $i>k$ one can replace $(k+i+1)$ by $(i+1)$ and one obtains

$$
\sum_{i=k+1}^{\infty} A_{i}^{[s]-s} r^{4(k+i)}(k+i+1)^{s-[s+1]+l} \leq C r^{4 k} \begin{cases}r^{4(k+1)}\left((k+1)\left(1-r^{4 k}\right)\right)^{-1 / 2}, l=0 \\ r^{4(k+1)}\left(1-r^{4 k}\right)^{-l} & , l \neq 0\end{cases}
$$

hence

$$
\sum_{k=0}^{\infty} A_{k}^{\alpha+s}\left|r^{2 \alpha+2} m_{k} \Delta^{s}\left(\lambda_{k}^{s} r^{4 k}\right)\right|^{2} \leq C r^{4 \alpha+4}(1-r)^{-\alpha-s-1}\|m\|_{2, s}^{2} .
$$

One should note that in these estimates essentially $s>\alpha+1 \geq 1 / 2$ is used. The contribution of the error term is estimated analogously. 
Let us conclude with estimating the terms which require smoothness of the multiplier sequence in question. Observe that $(k+1+i) \approx(k+1)$ if $0 \leq i<s$. Then

$$
\begin{gathered}
\sum_{k=1}^{\infty}\left|r^{2 \alpha+2} \Delta^{i}\left(\lambda_{k}^{s} r^{4 k}\right) \Delta^{s-i} m_{k+i}\right|^{2} \\
\leq C \sum_{n=1}^{\infty} \sum_{k=2^{n-1}}^{2^{n}-1}(k+1)^{\alpha+s} \sum_{l=0}^{i}(1-r)^{2 l} r^{8 k}(k+1)^{2 l+1}\left|(k+i)^{s-i} \Delta^{s-i} m_{k+i}\right|^{2}(k+i)^{-1} \\
\leq C \sum_{l=0}^{i}(1-r)^{2 l}\|m\|_{2, s-i}^{2} \sum_{n=1}^{\infty} 2^{n(\alpha+s+2 l+1)} r^{42^{n}} \\
\leq C\|m\|_{2, s-i}^{2}(1-r)^{-\alpha-s-1} \leq C\|m\|_{2, s}^{2}(1-r)^{-\alpha-s-1}
\end{gathered}
$$

again by the embedding properties of the $w b v$-spaces.

Thus Proposition 3.1 holds and Theorem 1.1 is established.

\section{A weighted transplantation theorem.}

In this context it is convenient to work with the Laguerre functions $\left\{\mathcal{L}_{k}^{\alpha}\right\}$, introduced in (2) at the beginning. The following transplantation theorem for Laguerre function expansions has recently been proved by Kanjin [10].

Theorem A. Let $\alpha, \beta>-1$ and $\varepsilon=\min \{\alpha, \beta\}$. If $\varepsilon \geq 0$, then

$$
\left\|\sum b_{k} \mathcal{L}_{k}^{\alpha}\right\|_{L^{p}\left(\mathbf{R}_{+}, d x\right)} \leq C\left\|\sum b_{k} \mathcal{L}_{k}^{\beta}\right\|_{L^{p}\left(\mathbf{R}_{+}, d x\right)}
$$

for $1<p<\infty$, where $C$ is a constant independent of $f$. If $-1<\varepsilon<0$, then the assertion remains true provided $p$ satisfies $(1+\varepsilon / 2)^{-1}<p<-2 / \varepsilon$.

Thangavelu [24] gave a modification of Kanjin's result by replacing the Lebesgue measure $d x$ by $x^{p / 4-1 / 2} d x$ (under the assumption $\varepsilon \geq-1 / 2$ ). Here we admit more general power weights.

Theorem 4.1 Let $1<p<\infty, \alpha, \beta>-1$ and $\varepsilon=\min \{\alpha, \beta\}$. If $\varepsilon \geq 0$, then

$$
\left\|\sum b_{k} \mathcal{L}_{k}^{\alpha}\right\|_{L_{v(\delta)}^{p}} \leq C\left\|\sum b_{k} \mathcal{L}_{k}^{\beta}\right\|_{L_{v(\delta)}^{p}}
$$

for $-1<\delta<p-1$, where $C$ is a constant independent of $f$. If $-1<\varepsilon<0$, then (13) holds for $-1-\varepsilon p / 2<\delta<p-1+\varepsilon p / 2$. 
Transplantation results were proved for various orthogonal expansions (cf. [10] for a brief exposition).

Proof. Looking at Kanjin's proof one discerns two lines in the argumentation.

The first one consists in pointwise reformulations, estimates, and tools like the projection formula

$$
L_{k}^{\mu+\nu}(x)=\frac{\Gamma(k+\mu+\nu+1)}{\Gamma(\nu) \Gamma(k+\mu+1)} \int_{0}^{1} y^{\mu}(1-y)^{\nu-1} L_{k}^{\mu}(y x) d y,
$$

$\operatorname{Re} \mu>-1, \operatorname{Re} \nu>0$, discussion of the smoothness properties of the involved "adjusting" multiplier sequences, verification of the Calderon-Zygmund property of a kernel, etc.

The second one concerns norm estimates. While Stein's interpolation theorem for analytic families of operators and a multiplier criterion of Butzer, Nessel, and Trebels [1] do not depend on a particular norm (as long as the hypotheses of these theorems are satisfied), there is a dependance of the norm in the case of the Calderon-Zygmund theory, Hardy's inequality, and Długosz' multiplier theorem.

If we now follow Kanjin's proof we only have to pay attention to the norm estimates and provide the necessary substitutes. We refer continuously to the notation used in [10]. For instance, by $M$ we will denote an admissible function, i.e. a positive function $M(\theta),-\infty<\theta<\infty$, that satisfies

$$
\sup _{\theta \in \mathbf{R}} e^{-a|\theta|} \log M(2 \theta)<\infty
$$

with some $0<a<\pi$. Also $\varphi(\theta)=\left\{\varphi_{n}(\theta)\right\}, \theta \in \mathbf{R}$, will denote the sequence defined by

$$
\varphi_{n}(\theta)=\left(\frac{\Gamma(n+\alpha+1)}{\Gamma(n+\alpha+1+i \theta)}\right)^{1 / 2} ;
$$

the "adjusting" operator $T_{\alpha, \varphi(\theta)}^{\beta}$ and the transplantation operator $T_{\alpha}^{\beta}$ are given by

$$
T_{\alpha, \varphi(\theta)}^{\beta} f \sim \sum \varphi_{n}(\theta)\left\langle f, \mathcal{L}_{n}^{\beta}\right\rangle \mathcal{L}_{n}^{\alpha}, \quad T_{\alpha}^{\beta}=T_{\alpha, \varphi(0)}^{\beta}
$$

where $\langle$,$\rangle stands for the usual scalar product in L^{2}\left(\mathbf{R}_{+}, d x\right)$.

Let us begin with the case $\alpha, \beta \geq 0$, temporarily assuming $\max \{-p / 2,-1\}<\delta<$ $\min \{p / 2, p-1\}$.

After some preliminary reductions, similar to those in [10], it is easy to see that the following proposition (cf. [10, Proposition 2]) is sufficient to prove Theorem 4.1.

Proposition 4.2 Let $\alpha \geq 0,1<p<\infty$, and $k=0,2$. Then

$$
\left\|T_{\alpha, \varphi(\theta)}^{\alpha+k+i \theta} f\right\|_{L_{v(\delta)}^{p}} \leq M(\theta)\|f\|_{L_{v(\delta)}^{p}}
$$

for all $\delta$ satisfying $\max \{-p / 2,-1\}<\delta<\min \{p / 2, p-1\}$ with an admissible $M$ independently of $f \in C_{c}^{\infty}$. 
To see, for instance, how (14) implies a weighted analogon of [10, Proposition 1,I] note that $\left\{\left(\varphi_{n}(\theta)\right)^{-1}\right\} \in w b v_{2, s}, s>\alpha+1$, whatever $\alpha \geq 0$ is and, moreover,

$$
\left\|\left\{\left(\varphi_{n}(\theta)\right)^{-1}\right\}\right\|_{2,[\alpha+2]} \leq C\left(1+|\theta|^{[\alpha+2]}\right)
$$

with $C$ independent of $\theta$. This follows from the calculus for the $w b v$-spaces [4] since by [10, Lemma 2]

$$
\sup _{x>0}\left|x^{j} \frac{d^{j}}{d x^{j}}\left(\frac{\Gamma(x+\alpha+1 / 2+i \theta)}{\Gamma(x+\alpha+1 / 2)}\right)^{1 / 2}\right| \leq C(\alpha, j)\left(1+|\theta|^{j}\right), \quad j \in \mathbf{N}_{0}, \alpha>-1 / 2,
$$

with $C$ independent of $\theta$. Therefore, by combining (3) and Theorem 1.1,

$$
\begin{gathered}
\left\|T_{\alpha}^{\alpha+k+i \theta} f\right\|_{L_{v(\delta)}^{p}}=\left\|\sum\left\langle f, \mathcal{L}_{n}^{\alpha+k+i \theta}\right\rangle \mathcal{L}_{n}^{\alpha}\right\|_{L_{v(\delta)}^{p}} \\
\leq C\left(1+|\theta|^{[\alpha+2]}\right)\left\|T_{\alpha, \varphi(\theta)}^{\alpha+k+i \theta} f\right\|_{L_{v(\delta)}^{p}} \leq M(\theta)\|f\|_{L_{v(\delta)}^{p}}
\end{gathered}
$$

provided (14) holds.

Proof of (14). To estimate $\left\|T_{\alpha, \varphi(\theta)}^{\alpha+i \theta} f\right\|_{L_{v(\delta)}^{p}}$ we just follow line by line Section 3 of 10 . making use in appropriate places of weighted Hardy's inequality

$$
\left(\int_{0}^{\infty}\left(\int_{x}^{\infty} f(y) d y\right)^{p} x^{\delta} d x\right)^{1 / p} \leq \frac{p}{\delta+1}\left(\int_{0}^{\infty}(y f(y))^{p} y^{\delta} d y\right)^{1 / p}
$$

valid for $f \geq 0$ and $\delta>-1$ (cf. [18, p. 272]) and of weighted inequality for singular integral operators. Recall that the interval $(-1, p-1)$ characterizes those $\delta$ 's for which the function $|x|^{\delta}$ belongs to $A_{p}(\mathbf{R})$. Therefore we can apply weighted singular integral inequalities.

Exactly the same means are used to estimate $\left\|T_{\alpha, \varphi(\theta)}^{\alpha+2+i \theta} f\right\|_{L_{v(\delta)}^{p}}$, cf. Section 4 of Kanjin's paper, except for the fact that we also need a weighted version of [10, (4.1)], i.e., the inequality

$$
\left\|M_{\Lambda}^{\alpha}(f)\right\|_{L_{v(\delta)}^{p}} \leq C\|\Lambda\|_{b q c}\|f\|_{L_{v(\delta)}^{p}} .
$$

Here one can apply a result of Poiani [16, Corollary, p. 11], which gives the uniform boundedness of the Cesàro means of order 1 on weighted $L^{p}\left(x^{\delta} d x\right)$-space, $-1<\delta<$ $p-1$, that is needed in the assumptions in Butzer, Nessel, and Trebels [ [1, Theorem $3.2]$, and one concludes the validity of (15).

This finishes an outline of necessary changes in Kanjin's proof that have to be done in order to prove the weighted transplantation result in the case $\alpha, \beta \geq 0$ and $\max \{-p / 2,-1\}<\delta<\min \{p / 2, p-1\}$. 
To settle the open cases let us look back at the previous outline and assume without loss of generality that $\alpha<\beta$. The restrictions on $\alpha, \beta$ as well as on $\delta$ were caused by the application of Theorem 1.1. For the proof of (14) in the case $k=2$ and $\theta=0$,

$$
\left\|T_{\alpha}^{\alpha+2} f\right\|_{L_{v(\delta)}^{p}} \leq C\|f\|_{L_{v(\delta)}^{p}},
$$

Theorem 1.1 is not needed and a restriction can only come into play by the quasiconvexity criterion, i.e. by Poiani's [16, Corollary, p. 11] result which implies in our case, if $\alpha<0$, that $-1-\alpha p / 2<\delta<p-1+\alpha p / 2$, hence (16) holds for these $\delta$ 's.

On the other hand, Theorem 1.1 is true for $-1<\delta=\gamma-\alpha<p-1$ if $\alpha$ is sufficiently large, say $\alpha>A>0$ so that no restriction on $\delta$ happens in the transplantation theorem if $\alpha, \beta \geq A$. If we choose $N \in \mathbf{N}_{0}$ so large that $\alpha+2 N \geq A$, the transplantation theorem proved so far, gives

$$
\left\|T_{\alpha+2 N}^{\beta+2 N} f\right\|_{L_{v(\delta)}^{p}} \leq C\|f\|_{L_{v(\delta)}^{p}} .
$$

The rest of the assertion now follows by (16), (17), duality, the semigroup property of $T_{\alpha}^{\beta}$ (see [10]) from

$$
T_{\alpha}^{\beta}=T_{\alpha}^{\alpha+2} \circ \cdots \circ T_{\alpha+2 N-2}^{\alpha+2 N} \circ T_{\alpha+2 N}^{\beta+2 N} \circ T_{\beta+2 N}^{\beta+2 N-2} \circ \cdots \circ T_{\beta+2}^{\beta} .
$$

Hence, Theorem 4.1 is completely established.

An immediate consequence of Theorem 4.1 in combination with (3) yields for Laguerre multipliers

Corollary 4.3 Let $\alpha, p, \delta$ be as in Theorem 4.1. Then

$$
M_{\alpha, \alpha p / 2+\delta}^{p}=\mathcal{M}_{\alpha, \delta}^{p}=\mathcal{M}_{0, \delta}^{p}=M_{0, \delta}^{p} .
$$

Remarks. 1) For $\delta=0$ the formula (18) is just Kanjin's [10] multiplier result, while for $\delta=p / 4-1 / 2$ we cover Thangavelu's [24] statement on multipliers.

2) If we choose $\alpha=0$ and $p=2$ then $M_{\alpha, \alpha p / 2}^{p}$ and $M_{\alpha, \alpha p / 2+p / 4-1 / 2}^{p}$ coincide with $M_{0,0}^{2}=l^{\infty}$. Since $w b v_{2, s_{0}} \subset l^{\infty}, s_{0}>1 / 2$, interpolation between Theorem 1.1 for $\alpha=0, p>1, p \approx 1$ and $w b v_{2, s_{0}} \subset M_{0,0}^{2}$ along the lines of [2] yields the following improvement of the the sufficient multiplier criteria given in [10] and [24]:

Corollary 4.4 Let $\alpha>-1$, then

$$
w b v_{2, s} \subset M_{\alpha, \alpha p / 2}^{p}, \quad w b v_{2, s} \subset M_{\alpha, \alpha p / 2+p / 4-1 / 2}^{p}, \quad s>\max \{1 / p, 1-1 / p\},
$$


provided $p$ and $\delta$ satisfy the conditions of Theorem 4.1 where $\delta=0$ and $\delta=p / 4-1 / 2$, resp.

In particular, if $m(x)$ is a bounded one time differentiable function on $\mathbf{R}_{+}$satisfying

$$
\sup _{x}|m(x)|^{2}+\sup _{N} \int_{N}^{2 N}\left|m^{\prime}(x)\right|^{2} x d x \leq B^{2},
$$

then, if we set $m_{k}=m(k)$, there holds

$$
\left\|T_{m} f\right\|_{L_{v(\alpha p / 2)}^{p}} \leq C B\|f\|_{L_{v(\alpha p / 2)}^{p}}, \quad\left\|T_{m} f\right\|_{L_{v(\alpha p / 2+p / 4-1 / 2)}^{p}} \leq C B\|f\|_{L_{v(\alpha p / 2+p / 4-1 / 2)}^{p}} .
$$

3) To prove (5) we choose in (3) $\gamma=\alpha p(1 / p-1 / 2)$. By the transplantation Theorem 4.1 we obtain

$$
M_{\alpha, \alpha}^{p}=\mathcal{M}_{\alpha, \alpha p(1 / p-1 / 2)}^{p}=\mathcal{M}_{0, \alpha p(1 / p-1 / 2)}^{p} \supset w b v_{2, s_{0}}, \quad s_{0}>1,
$$

where the last inclusion follows from Theorem 1.1 provided $\max \{-p / 2,-1\}<\alpha p(1 / p-$ $1 / 2)<\min \{p / 2, p-1\}$. But the latter right inequality leads to a restriction on $p, p \leq 2$, namely

$$
\alpha p(1 / p-1 / 2)<p-1 \Longleftrightarrow \frac{2 \alpha+2}{\alpha+2}<p \leq 2,
$$

thus, by duality, (5) is established.

4) Interpolation between $w b v_{2, s_{0}} \subset M_{\alpha, \alpha}^{p}, s_{0}>1, \alpha \geq 0$, with $p$ as in the preceding remark and $l^{\infty} \subset M_{\alpha, \alpha}^{2}$ leads to

$$
w b v_{q, s} \subset M_{\alpha, \alpha}^{p}, \quad s>(2 \alpha+2)|1 / p-1 / 2|, \quad q<2 / s, \quad \frac{2 \alpha+2}{\alpha+2}<p<\frac{2 \alpha+2}{\alpha} .
$$

If one applies Corollary 1.2 and the latter criterion upon the multiplier sequence $m_{\zeta, \eta}=\left\{m_{\zeta, \eta}(k)\right\}, m_{\zeta, \eta}(k)=k^{-\zeta \eta} \exp \left(i k^{\eta}\right), \quad k \neq 0, \quad \eta>0$, one obtains $m_{\zeta, \eta} \in$ $M_{\alpha, \alpha}^{p}, \alpha \geq 0$, provided $\zeta>(2 \alpha+2)|1 / p-1 / 2|$.

5) Let us conclude with a multiplier criterion for $M_{\alpha, \alpha}^{p}$ in the case $-1<\alpha<0$. Recalling (19), observing $-1-\alpha p / 2<\alpha-\alpha p / 2<p-1+\alpha p / 2$ for all $p, 1<p<\infty$, and using (20) in the case $\alpha=0$, Theorem $4.1(\beta=0)$ yields

Corollary 4.5 Let $-1<\alpha<0,1<p<\infty$. Then

$$
w b v_{q, s} \subset M_{\alpha, \alpha}^{p}, \quad s>2|1 / p-1 / 2|, \quad 1 / q>|1 / p-1 / 2| .
$$

This may be looked at as a weak supplement to Corollary 1.2 a) in so far as, if one considers the Cesàro means of order $\nu$ in the case $\alpha=-1 / 2$ (cf. the discussion to Corollary 1.2), one only obtains uniform boundedness if $\nu>1 / 4$ for $p>4 / 3, p$ near 4/3, whereas Muckenhoupt [14] has shown in this instance even the uniform boundedness of the partial sums $(\nu=0)$. 


\section{References}

[1] P.L. Butzer, R.J. Nessel, And W. Trebels, On summation processes of Fourier expansions in Banach spaces I, Tohoku Math. J., 24 (1972), 127 - 140.

[2] W. C. Connett And A. L. Schwartz, The theory of ultraspherical multipliers, Mem. Amer. Math. Soc. 183 (1977).

[3] J. Deugosz, $L^{p}$-multipliers for the Laguerre expansions, Colloq. Math. 54 (1987), pp. $287-293$.

[4] G. Gasper and W. Trebels, A characterization of localized Bessel potential spaces and applications to Jacobi and Hankel multipliers, Studia Math., 65 (1979), pp. $243-278$.

[5] G. Gasper and W. Trebels, Multiplier criteria of Hörmander type for Jacobi expansions, Studia Math., 68 (1980), pp. 187 -197.

[6] G. Gasper and W. Trebels, A Hausdorff-Young inequality and necessary conditions for Jacobi expansions, Acta Sci. Math. (Szeged), 42 (1980), pp. 247 -255 .

[7] G. Gasper and W. Trebels, Necessary multiplier conditions for Laguerre expansions, Canad. J. Math., 43 (1991), 1228 - 1242; II, SIAM J. Math. Anal. (to appear).

[8] E. Görlich and C. Markett, A convolution structure for Laguerre series, Indag. Math., 44 (1982), pp. $161-171$.

[9] I.I. Hirschman, The decomposition of Walsh and Fourier series, Mem. Amer. Math. Soc. no. 15 (1955).

[10] Y. Kanjin, A transplantation theorem for Laguerre series, Tohoku Math. J. 43 (1991), pp. $537-555$.

[11] C. Markett, Mean Cesàro summability of Laguerre expansions and norm estimates with shifted parameter, Anal. Math., 8 (1982), pp. 19 - 37.

[12] C. Markett, Cohen type inequalities for Jacobi, Laguerre and Hermite expansions, SIAM J. Math. Anal., 14 (1983), pp. 819 - 833.

[13] S. Meda, A general multiplier theorem, Proc. Amer. Math. Soc., 110 (1990), pp. $639-647$.

[14] B. Muckenhoupt, Mean convergence of Hermite and Laguerre series, II, Trans. Amer. Math. Soc., 147 (1970), pp. 433 - 460. 
[15] A. PeyerimhofF, Über Summierbarkeitsfaktoren und verwandte Fragen bei Cesàroverfahren, II Acad. Serbe Sci., Publ. Inst. Math., 10 (1956), pp. 1 - 18.

[16] E.L. Poinni, Mean Cesàro summability of Laguerre and Hermite series, Trans. Amer. Math. Soc., 173 (1972), 1 - 31.

[17] E.M. Stein, Note on singular integrals, Proc. Amer. Math. Soc., 8 (1957), pp. $250-254$.

[18] E.M. Stein, Singular Integrals and Differentiability Properties of Functions, Princeton Univ. Press, Princeton, N.J., 1970.

[19] E.M. STEIN AND G. WeISS, Interpolation of operators with change of measures,Trans. Amer. Math. Soc., 87 (1958), pp. 159 - 172.

[20] K. Stempak, Almost everywhere summability of Laguerre series, Studia Math., 100 (1991), pp. 129 - 147; II, ibid. 103 (1992), pp. 317 - 327.

[21] K. Stempak, Heat diffusion and Poisson integrals for Laguerre expansions, Tohoku Math. J. (to appear).

[22] R.S. Strichartz, Multipliers for spherical harmonic expansions, Trans. Amer. Math. Soc., 167 (1972), pp. 115 - 124.

[23] S. Thangavelu, Littlewood-Paley-Stein theory on $\mathbf{C}^{n}$ and Weyl multipliers, Revist. Mat. Ibero., 6 (1990), pp. 75 - 90.

[24] S. Thangavelu, Transplantation, summability and multipliers for multiple Laguerre expansions, Tohoku Math. J., 44 (1992), pp. 279 - 298.

[25] G. Szegö, Orthogonal Polynomials, 4th ed., Amer. Math. Soc. Colloq. Publ. 23, Providence, R.I., 1975. 